\title{
Implementation of Ultra Low Power Gadget for Mobile Phone
}

\author{
Prajakta S. Chavan \\ Electronics Technology \\ Department of Technology \\ Shivaji University, Kolhapur \\ Maharashtra, India.
}

\author{
Uttam L.Bombale \\ Electronics Technology \\ Department of Technology \\ Shivaji University, Kolhapur \\ Maharashtra, India.
}

\begin{abstract}
The increasing popularity of communication has ushered in concern over security-related issues. Today's Smartphone and tablet device are very costly ranging from 300 USD to 600 USD, to avoid them to get forget at any social places, we can have gazette which will warn us (beep)this device is left back .To make commercialize product which will act as mobile watchmen, with specification and characteristic like smallest device foot print, comfortable to carry along, ultra low power consumption to increase battery life and to avoid frequent charging, ultra low Sleep currents and efficient performance. As per this specification we will develop a mobile phone watchman gadget which will connect to mobile phone by Bluetooth connectivity if this gadget will go beyond 4 to 6 meters gadget will beep loud when Bluetooth connectivity breaks
\end{abstract}

\section{Keywords}

Bluetooth low energy V4.0, BT Protocol, CC2540.

\section{INTRODUCTION}

Increasing popularity of communication has ushered in concern over security-related issues. Many times we forget to pickup our mobile phone from restaurant, library, canteen and other social places. Probability for device stolen from this place is also high. When we realize the portable device mobile, tablet or laptop is left back at restoring its quite late and device is pickup or stole by stranger, this smart phone and tablet device are very costly. According to crime statistics on mobile stolen cases from 2001 to 2013 graph goes on increasing and it is very difficult to search that mobile device like laptops, cell phones, tablets. So precaution is good than cure i.e. we must keep our mobile device from any place, but there is chance to forget our mobile device at any place. To avoid them to get forget at any social places, we can have gazette which will warn us (beep) this device is left back.

According to the objective of project, we develop hardware board for ultra low power microcontroller system which is the heart of the system and control the entire system then search from the various Bluetooth module systems and choose appropriate module that for our system CC2540 is the $2.4 \mathrm{GHz}$ Bluetooth low energy system-on-chip, which is having 8051 with Bluetooth stack and can run both applications and Bluetooth low energy protocol stack. [1-2]

\section{DESIGN METHOD OF GADGET}

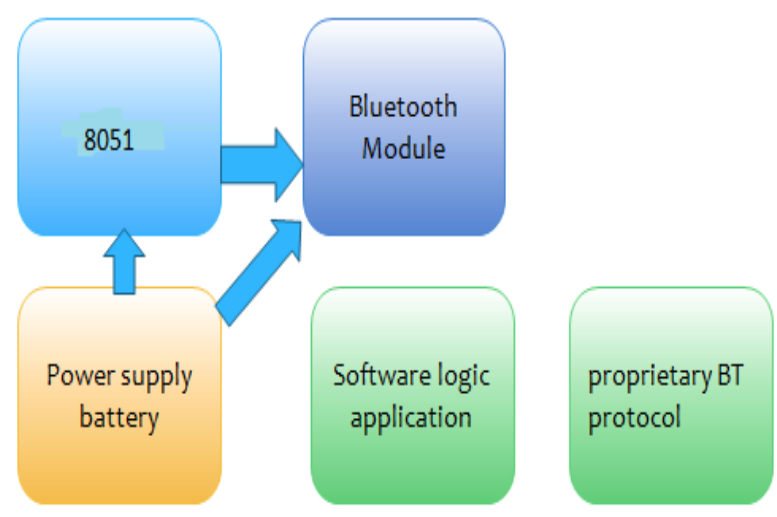

Fig 1: Block diagram of gadget

True Single-Chip BLE Solution CC2540 can run Both Application and BLE Protocol Stack includes Peripherals to Interface with wide range of Sensors. The CC2540 is a costeffective, low-power, true system-on-chip (SoC) for Bluetooth low energy applications. It enables robust BLE master or slave nodes to be built with very low total bill-of-material costs.[3] The CC2540 combines an excellent RF transceiver with an industry-standard enhanced $8051 \mathrm{MCU}$, in-system programmable flash memory, 8-KB RAM, and many other powerful supporting features and peripherals. The CC2540 is suitable for systems where very low power consumption is required. Very low-power sleep modes are available. Short transition times between operating modes further enable low power consumption. [4] The CC2540 comes in two different versions CC2540F128/F256, with 128 and $256 \mathrm{~KB}$ of flash memory, respectively. Combined with the Bluetooth low energy protocol stack, the CC2540F128/F256 forms the market's most flexible and cost-effective single-mode. [5]

Bluetooth low energy solution. Version 4.0 of the Bluetooth standard allows for two systems of wireless technology: Basic Rate (BR; often referred to as "BR/EDR" for "Basic Rate / Enhanced Data Rate") and Bluetooth low energy (BLE). The BLE system was created for the purpose of transmitting very small packets of data at a time, while consuming significantly less power than BR/EDR devices.

Devices that can support BR and BLE are referred to as dualmode devices and go under the branding Bluetooth Smart Ready. Typically in a Bluetooth system, a mobile phone or laptop computer will be a dual-mode device. Devices that only support BLE are referred to as single-mode devices and go under the branding Bluetooth Smart. These single-mode 
devices are generally used for application in which low power consumption is a primary concern, such as those that run on coin cell batteries.

The BLE protocol stack consists of two sections: the controller and the host. This separation of controller and host goes back to standard Bluetooth BR/EDR devices, in which the two sections were often implemented separately. Any profiles and applications that are being used sit on top of the Generic Access Profile (GAP) and Generic Attribute Profile (GATT) layers of the stack.

The controller, host, profiles, and application are all implemented on the CC2540 as a true single chip solution. This is the simplest and most common configuration when using the CC2540.It is most cost effective and provides the lowest-power performance.[6-9]

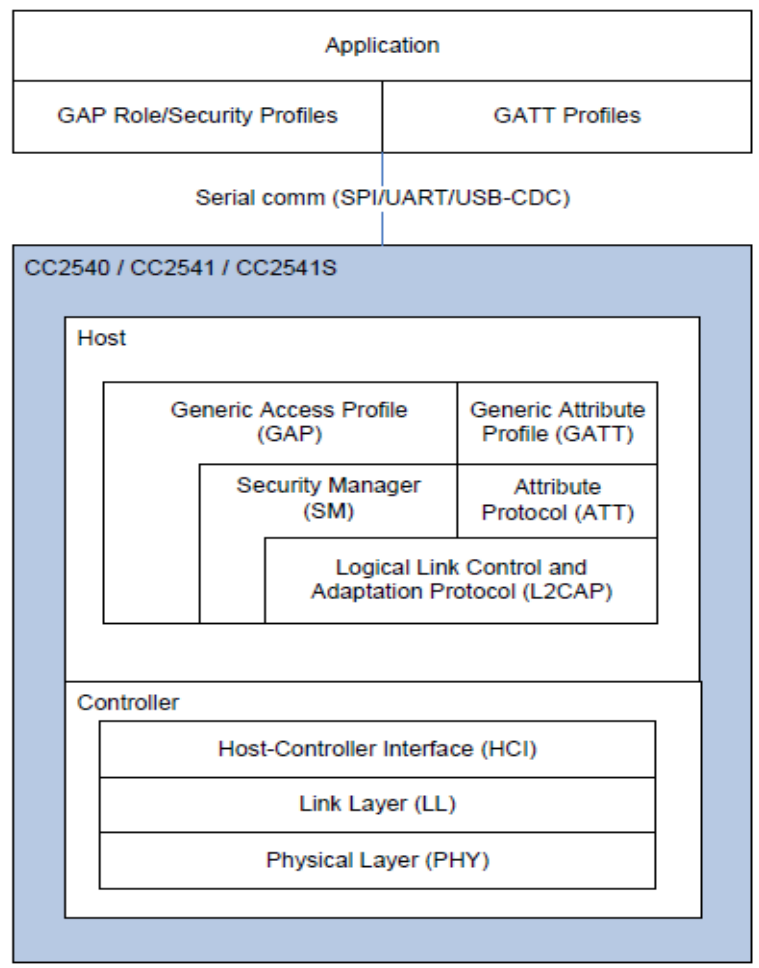

Fig 2: Network processors configuration

\section{WORKING WITH IAR EMBEDDED WORKBENCH}

This IAR system provides a complete range of development tools for embedded systems, C/C++ compilers, debuggers, state machine tools, development kits and RTOS. All embedded software for the CC2540 is developed using Embedded Workbench for 80518.10 .4 from IAR Software. After installing IAR Embedded Workbench, be sure to download all of the latest patches from IAR, as they will be required in order to build and debug projects with the CC2540.

Once all of the patches have been installed, develop the software for the CC2540/41. This section provides information on where to find this software. It also contains some basics on the usage of IAR, such as opening and building projects, as well as information on the configuration of projects using the BLE protocol stack. IAR contains many features that go beyond the scope of this document. [10]
This will compile the source code, link the files, and build the project. Any compiler errors or warnings will appear in the messages window at the bottom of the screen. To download the compiled code into a CC2540 device and debug, connect the gadget using a hardware debugger (such as the CC Debugger) connected to the PC over USB. Find the "Debug" button in the upper right side of the IAR window. Once the code is downloaded, a toolbar with the debug commands will appear in the upper left corner of the screen. Then start the program's execution by pressing the "Go" button on the toolbar. Once the program is running, we can get out of the debugging mode by pressing the "Stop Debugging" button.

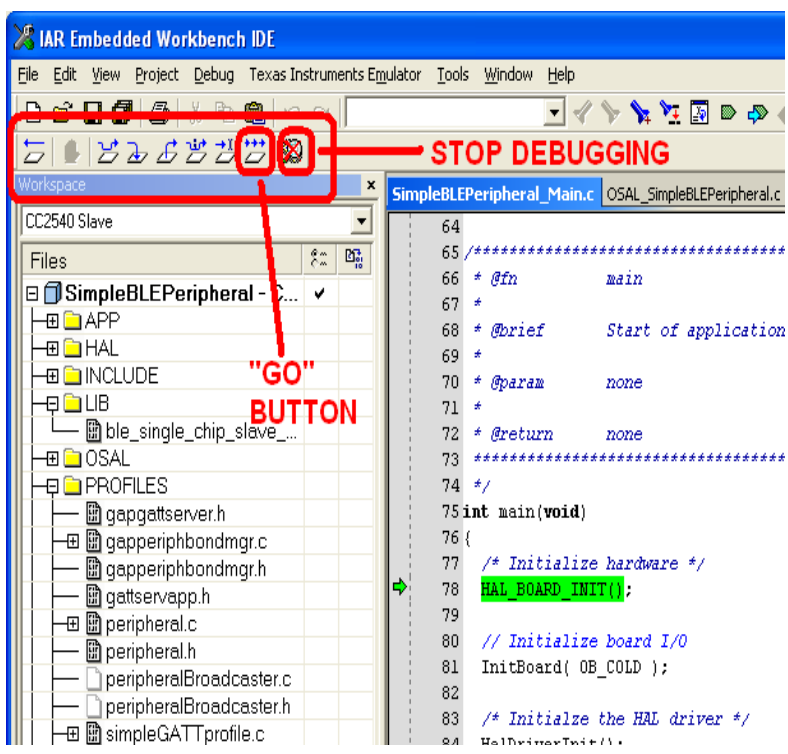

Fig 3: IAR Embedded workbench compile window

Then download the code in CC2540 using CC debugger. At this point the program should be executing on its own. The hardware debugger can be disconnected from the CC2540/41 and will continue to run as long as the device remains powered-up.

\subsection{Mobile phone application development}

Gadget should connect to mobile phone which is having Bluetooth version 4.0 that is Bluetooth low energy. So develop the Application using JAVA eclipse. Develop android code using android SDK tool v22.6 in eclipse Integrated development environment. Run program with API 18.

Using this Application we can connect mobile phone with gadget using Bluetooth connection.[11-12]

\section{EXPERIMENTAL RESULT}

Proposed work is satisfactory work with all Bluetooth low energy mobile phone.

\subsection{Hardware Setup}

With CC2540 peripherals required for our gadget

1. Dual coloured Light Emitting Diode(LED)

2. Switch buttons(SW1,SW2)

3. Buzzer

With the LED on gadget gives the status of mobile phone or remote device connected or not. Using two switch buttons on Gadget one is used for advertise the gadget when scan with mobile phone and another button is used for disconnect the gadget with phone. Following figure is the hardware of 
gadget. There are two 8 pin adaptors used in gadget one is for debug and burn the code in gadget and another is for testing the future modified code.

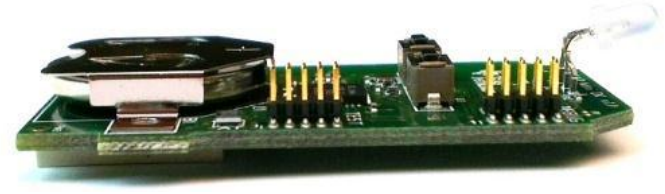

Fig 4: Hardware of gadget

First, need to power up the CC2540 and insert the CR 2032 battery then LED will be light green for one second.

Toggle Advertisements on and off by pushing the right button on the CC2540.During advertisement, the LED will be blinking red. Connect mobile phone with gadget using the application on phone.

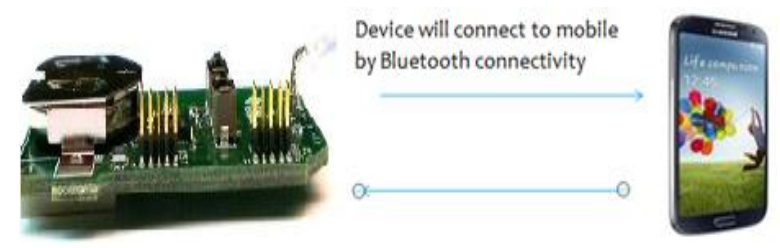

Fig 5: Gadget-mobile phone connection

To sound the buzzer located on the CC2540, writing the code value in the CC2540 code in the IAR Embedded workbench following value

\section{$\square$ 01:00 for low Alert \\ $\square$ 02:00 for high Alert}

$\square$ 00:00 to turn off.

So the buzzer will sound for 10 seconds when Bluetooth connectivity breaks with mobile phone.

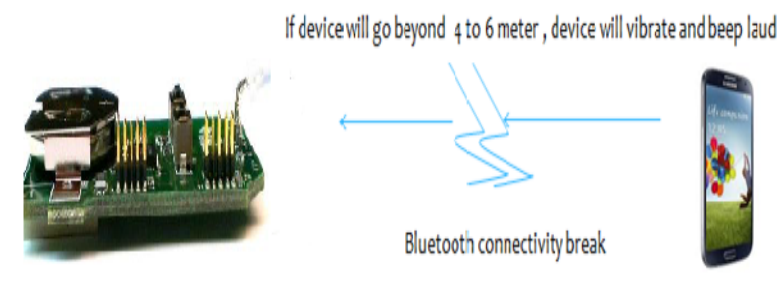

Fig 6: Gadget-mobile phone disconnect

Following are the LED status of gadget when connects and disconnect with the mobile phone.

Table 1: LED status of gadget

\begin{tabular}{|l|l|}
\hline LED action & Status \\
\hline Solid red & Ideal \\
\hline Blinking red & Scanning for any BLE remote \\
\hline Solid green & Connected \\
\hline $\begin{array}{l}\text { Blinking } \\
\text { green }\end{array}$ & $\begin{array}{l}\text { Scanning for previously bonded } \\
\text { remote }\end{array}$ \\
\hline
\end{tabular}

For connecting mobile phone with gadget there two switches on gadget. Following are the states and corresponding actions performed with switch buttons.

Table 2: Switch button action of gadget

\begin{tabular}{|l|l|l|}
\hline Switch button & State & Action \\
\hline SW1 & Ideal & $\begin{array}{l}\text { Erase bonding } \\
\text { action }\end{array}$ \\
\hline SW2 & Ideal & Start scanning \\
\hline
\end{tabular}

\section{CONCLUTION}

All methods until now made for the devices which are stolen by strangers, that is after misplacing the device like, tracking. But for that device should be continually connected with internet and this is not reliable process. This project name as mobile watchman device work as really as a watchman for our devices like mobile phones, tablets, laptops, and don't misplace the device is better than searching the device after misplacing. So this project is very important.

It also has future work like make the product size variable as small as placed in wrist watch. So the cost decreases. Add more functionality in the product such as add display in the device for display messages from mobile phone and display current location of device. Add up to 16 mobile devices like phone, laptop, and tablet at a time, and connect all to mobile gadget.

\section{REFERENCES}

[1] C. Piguet, et al.,"Low-power low-voltage microcontrollers: Architectures and performances comparison", Low-Power Low-Voltage Workshop at ESSCIRC',96, 1996

[2] Piguet,C. Centre Suisse d"Electronique et de Microtechnique SA, Neuchatel, Switzerland "Low-power design of 8-b embedded CoolRisc microcontroller cores" Solid-State Circuits, IEEE Journal of (Volume:32, Issue: 7 ) 06 August 2002, 0018-9200

[3] Bluetooth ${ }^{\circledR}$ Core Technical Specification document, version 4.0 online available at http://www.bluetooth.com/SiteCollectionDocumets/Core _V40.zip

[4] CC253x System-on-Chip Solution for 2.4-GHz IEEE 802.15.4 and ZigBee® Applications/CC2540 System-onChip Solution for 2.4-GHz Bluetooth low energy Applications

[5] CC2540 controller datasheet for 2.4-GHz Bluetooth low energy System-on-chip online available at www.ti.com

[6] "Design of an ultra-low power device for aircraft structural health monitoring" Perelli, Alessandro ; Caione, Carlo ; De Marchi, Luca ; Brunelli, Davide ; Marzani, Alessandro; Benini, Luca Design, Automation \& Test in Europe Conference \& Exhibition(DATE) 2013 Digital Object Identifier: 10.7873/DATE.2013.236 Publication Year: 2013 , Page(s): 1127 - 1130

[7] "Practical Applications of Low-Power Design with NanoWatt XLPTM”. Mark Hofmann - Advanced Engineer, Future Electronics. 
[8] "Simplifications of the Bluetooth radio liudevices" Youquan Zheng ; Zhenming Feng Networked Appliances, 2002. Gaithersburg. Proceedings. 2002 IEEE 4th International Workshop on Digital Object Identifier: 10.1109/IWNA.2001.980816 Publication Year:2002,Page(s):107-115 Cited by: Papers(2) IEEE CONFERENCE PUBLICATIONS

[9] Morón, M.J. ;Luque, R. ;Casilari, E. ; Diaz-Estrella, A. "Characterization of bluetooth packet delay in noisy environments" ,IEEE Volume:13,Issue: DigitalObjectIdentifier:10.1109/LCOMM.2009.091142 Publication Year: 2009 , Page(s): 661 - 663 IEEE JOURNALS \& MAGAZINES
[10] IAR Embedded workbench software online available at www.iar.com/ew8051.

[11] "Performance analysis of an Bluetooth Low Energy sensor system" Mackensen, E. ; Lai, M.;Wendt,T.M.Wireless Systems (IDAACS-SWS), 2012 IEEE 1st International Symposium on Digital Object Identifier: $\quad$ 10.1109/IDAACS-SWS.2012.6377634 Publication Year: 2012 , Page(s): $62-66$

[12] "Bhagwat,p. "Bluetooth: technology for short-range wireless apps", IEEE Volume:5,Issue:3 Digital Object Identifier:10.1109/4236.935183 Publication Year:2001, Page(s):96-103 Cited by: Papers (14) | Patents (5) IEEE JOURNALS \& MAGAZINES 